\title{
FRAM for systemic accident analysis: a matrix representation of functional resonance
}

\author{
Patriarca Riccardo*1, Del Pinto Gianluca2 ${ }^{*}$ Di Gravio Giulio ${ }^{1}$, Costantino Francesco ${ }^{1}$ \\ ${ }^{1}$ Department of Mechanical and Aerospace Engineering, \\ Sapienza University of Rome \\ Via Eudossiana, 18 - 00184 Rome (Italy) \\ [riccardo.patriarca; giulio.digravio; francesco.costantino]@uniroma1.it \\ ${ }^{2} A N A C N A$ \\ Italian Air Traffic Controllers' Association \\ Via Camilla 39/41 00181 Rome (Italy) \\ delpintogianluca@gmail.com
}

*Corresponding author 


\title{
FRAM for systemic accident analysis: a matrix representation of functional resonance
}

\begin{abstract}
Due to the inherent complexity of nowadays Air Traffic Management (ATM) system, standard methods looking at an event as a linear sequence of failures might become inappropriate. For this purpose, adopting a systemic perspective, the Functional Resonance Analysis Method (FRAM) originally developed by Hollnagel, helps identifying non-linear combinations of events and interrelationships.

This paper aims to enhance the strength of FRAM-based accident analyses, discussing the Resilience Analysis Matrix (RAM), a user-friendly tool that supports the analyst during the analysis, in order to reduce the complexity of representation of FRAM. The RAM offers a two dimensional representation which highlights systematically connections among couplings, and thus even highly connected group of couplings. As an illustrative case study, this paper develops a systemic accident analysis for the runway incursion happened in February 1991 at LAX airport, involving SkyWest Flight 5569 and USAir Flight 1493. FRAM confirms itself a powerful method to characterize the variability of the operational scenario, identifying the dynamic couplings with a critical role during the event and helping discussing the systemic effects of variability at different level of analysis.
\end{abstract}

\section{KEYWORDS}

Accident analysis; FRAM; air traffic control; air traffic management; safety; resilience engineering; runway incursion; socio-technical system. 


\section{INTRODUCTION}

The traditional definition of safety as "a condition where nothing goes wrong or where the number of things that go wrong is acceptably small" has been widely adopted over years ${ }^{1}$. This definition, linked with the naturalness of linear thinking and the subsequent cause-effect relationship led to a well-established safety strategy: decomposing the system and describing it using as many details as possible to detect the causes and develop strategies to remove them or at least to minimize their effects. However, this perspective appeared to be progressively inaccurate to describe and analyze modern socio-technical systems. The causality credo and a linear cause-effect reasoning no longer represent universally valuable concepts. In particular, the complexity of current ATM system does not allow relying on individual reliability analysis, based on a bimodal nature of work, i.e. functioning and not functioning. System decomposability is often hardly possible to acquire, due to inherent flaws of decomposing tight-coupled functions. Systemic approaches become thus necessary, acknowledging the relevance of understanding the inherent complexity rather than simplifying it. In terms of safety analyses, rather than focusing only on adverse outcomes and trying to delve into the causes of system malfunctioning, it becomes necessary to understand system normal functioning. In line with this perspective, safety can be defined as "the ability to succeed under varying conditions, so that the number of intended and acceptable outcomes is as high as possible". This definition, in line with the Resilience Engineering theory, acquires a relevant role for the ATM systems, which are characterized by a large number of interacting human, organizational, technical aspects.

Furthermore, as shown by recent tendency in airport operations, by 203019 European important airports - among which will be Paris Charles De Gaulle, Warsaw, Athens, Wien, and Barcelona - will come to saturation. This congestion, combined with the tight-coupled nature of work at different human, technical and organization levels might generate losses both in terms of productivity, i.e. delays in operations, and expectable safety-related consequences ${ }^{2,3}$.

Following a more theoretical approach, it becomes necessary to look at safety, generally based on the so-called Safety-I perspective, also in terms of Safety-II. Safety-I relies on the following assumptions ${ }^{4}$ :

- $\quad$ Systems are decomposable and well-understood

- $\quad$ Systems and places of work are well-designed and correctly maintained

- $\quad$ Procedures are comprehensive, complete and correct

- $\quad$ Operators behave as they are expected to and as they have been trained to

- Designers have foreseen every contingency and have provided the system with appropriate response capabilities. 
On the contrary, for Safety-II:

- $\quad$ Systems cannot be decomposed in a meaningful way

- $\quad$ System functions are not bimodal but everyday performance is flexible and variable

- $\quad$ Human performance variability leads to success as well as failures

- $\quad$ Even though some outcome can be interpreted as linear consequences of other events, some event result of coupled performance variability.

Based on these assumptions, for reliable risk assessment and accident analyses, it becomes thus necessary to follow a systemic perspective, in line with Safety-II and Resilience Engineering. A reliable systemic accident analysis should go beyond advocating a human error as a cause, in order to understand non-linear functional effects of variability among agents in everyday operations. This perspective would allow focusing not only on individual failures, but also on systemic failures, acknowledging that accident might be the result of normal work in normal circumstances. Furthermore, the deviation (work-as-done) from the prescribed work (work-asimagined) might cause both positive and negative effects. Deviations have to be considered in agreement with the concept of local rationality, since in some circumstances, they become unavoidable and even necessary to deal with the complexity of the current scenario.

Following an operational perspective, the Functional Resonance Analysis Method (FRAM) developed by Hollnagel 1, offers a systemic perspective both for accident analysis and risk assessment, acknowledging the role of performance variability and inherent complexity.

This paper aims to discuss the role of FRAM for accident analysis, showing its benefits to describe the work-asdone in complex systems. Furthermore, considering the inherent limitation due to the FRAM's overwhelming complexity of representation, we developed a simple and user-friendly tool, i.e. the Resilience Analysis Matrix (RAM), enhancing a recently proposed approach ${ }^{5}$. The target of RAM consists of generating systematic assessments based on FRAM's systemic perspective, being a support tool for the traditional FRAM, in order to gain findings and ease a structured analysis of the system. The proposed structure of RAM enhances a traditional FRAM approach, offering detailed insights, even in combination with recent semi-quantitative evolutions of FRAM. This paper innovatively presents a detailed application of RAM in case of accident analysis.

The remainder of the paper is organized as follows. Section 2 describes relevant applications of FRAM in literature, with particular reference to accident analysis. Section 3 details the principles and building steps of an accident analysis based on FRAM, developing the theoretical foundation of RAM and discussing its potential benefits. Section 4 contextualizes these theoretical assumptions in the ATM system, with particular reference to runway 
incursions. Furthermore, Section 4 details an illustrative case study, based on the LAX airport accident, and happened the February 1,1991. The conclusion finally summarizes the good outcomes of the method and potential paths for future research.

\section{Evidence from literature}

The FRAM aims to model complex systems looking at their functional aspects rather than their physical structure, defining dynamic interactions among functions and modelling performance variability, which represents the source for both failures and successes. The FRAM allows thus a systemic representation of the system, in order to assess how variability might propagate through the system, potentially generating emerging risks.

When used for risk assessment, the FRAM aims at understanding how performance variability might affect other upstream and downstream functions, encouraging thoughts about how a potentially disruptive performance variability can be detected and monitored, or more classically dampened. For event investigation, the functional representation of the system is explored to understand how the an accident happened, and identify ways to manage performance variability more properly 1 .

Even though the FRAM is a quite recent method, it has been applied in different domains over years, (e.g.) for interpreting experimental results obtained from a railway simulator ${ }^{6}$, or to assess risks for nuclear power plants ${ }^{7}$ and process plants ${ }^{8}$. In the healthcare domain, FRAM has been applied to manage emerging risks emerging as a combination of human, organizational and technical variability within an emergency care pathway ${ }^{9}$, or for implementing clinical guidelines ${ }^{10}$, and for describing work-as-done in terms of system's resilience, in a case study about blood sampling activities ${ }^{11}$.

However, its first applications were in the aviation and the air traffic control: in 2007 a case study evaluated the effects of automation for the pilot cabin in order to understand system functioning in terms of deviations from operating procedures ${ }^{12}$, in relation to the flight 965 in Colombia happened in 1995. A FRAM-based accident analysis also lead to investigate the Alaska Airlines flight 261 crash into the Pacific Ocean, happened in 200013 . Studying the same accident, FRAM has been acknowledged to address five key characteristics, i.e. buffering capacity, flexibility, margin, tolerance, and cross-scale interactions ${ }^{14}$. Another accident analysis based on FRAM was related to the MD83 F-GRMC approaching Paris Orly airport in the 1997, which proved the relevance of functional resonance in terms of allowing an in-depth characterization of the evolving operating circumstances ${ }^{15}$. FRAM has been compared to traditional accident investigation also in the context of Comair flight 5191 of 2006, stating that the conclusions of the NTSB fall somewhat short of the mark addressing the crew error as a primary cause 16. The application unveiled complex interrelationships that would help defining appropriate 
countermeasures based on management performance variability rather than on simply constraining it. Similar outcomes arose from the mid-air collision between the B737-800 N600XL and an executive jet Embraer E-145, occurred in 2006 in the Amazonian sky ${ }^{17}$.

As a method, it has been compared to STEP in order to analyze the B737-36N NAX541 from Stavanger Sola airport to Oslo Gardermoen airport ${ }^{18}$. The analysis highlighted the relevance of FRAM to understand variability moving the analysis into the conditions of normal work, beyond the specifics of the time sequence and failure. Similar results emerged when comparing FRAM with fault tree analysis in an offshore operation accident: a system perspective unveils hidden factors leading to a comprehensive understanding of the accident itself ${ }^{19}$. It has been also confirmed 20 that FRAM has the potential to enrich hazard identification as a complementary tool, combined with Formal Safety Assessment (FSA), as emerged in a pilot study in the maritime domain.

Starting from incident data and normal operations, FRAM has been applied to define safety performance indicators for helicopter offshore ${ }^{21}$. In the study, the analysis of normal functioning and incident data was combined with interviews of different agents, i.e. helicopter pilots, ATCOs, helicopter deck operators and regulators. In addition, FRAM has been combined with different method and approaches, in order to overcome some limitations of the method itself. For example, it has been combined with HAMSTERS (Human-centered Assessment and Modelling to Support Task Engineering for Resilient System) and ICO (Interactive Cooperative Objects) to investigate the effects of automation in the ATM system ${ }^{22}$.

The FRAM has been also discussed in a semi-quantitative perspective, adopting Monte Carlo simulation to quantify the variability of couplings, showing a conceptual application for analyzing runway incursions ${ }^{23}$, industrial plant environmental risks ${ }^{24}$, or even emergent risks in space operations ${ }^{25}$. A model-checking-aided FRAM has also been developed to explore all the couplings among system functions and subsequently, to pinpoint critical sources of variability in everyday operations ${ }^{26}$. These approaches aim at developing more understandable results, rather than simply reducing the work-domain's complexity, isolating respectively critical paths and critical iterations of the model. Recent trends in the application of FRAM result thus in developing wider models, taking advantage of the systemic perspective of the method itself. However, in these cases the represented model are complicated and consequently present severe limitations in terms of comprehensibility of the results, due to their inherent highly interconnected structure 5 .

For this purpose, this paper shows the application of FRAM in case of an accident analysis in the aviation context, developing a FRAM model based on historic data and evaluations by Subject Matter Experts (SMEs). Besides the traditional four steps, starting from a recently proposed framework 5 , this paper suggests an enhanced 
representation of the system states, i.e. a matrix representation: the Resilience Analysis Matrix (RAM), an userfriendly representation that allows systematic analysis even for the more complicated FRAM models. A detail application of the RAM will show how to apply it in practice, following the building steps of the FRAM, according to a theoretical perspective and a detailed case study.

\section{Principles and building steps of FRAM}

FRAM relies on four principles ${ }^{1}$ :

- Equivalence of successes and failures: Successes and failures have the same origin, i.e. performance variability. In this sense, the FRAM is in line with Resilience Engineering perspective on failures. More specifically, it is possible to experience a failure due to the difficulties arising from adapting the system functioning to cope with real world, rather than consider failures as normal events on the system.

- Approximate adjustments: People as individuals or as a group and organizations adjust their everyday performance to cope with everyday operating conditions, resources and requirements. Due to resources finiteness, such adjustments appear to be approximate rather than exact.

- Emergence: Identifying a specific event (or a linear chain of events) as a cause of a system failure is a difficult, even impossible, task. Many events appear to be emergent rather than resultant from a specific combination of conditions, since the causal relationship is not always detectable.

- Functional Resonance: The functional resonance represents the detectable signal emerging from the unintended interaction of the everyday variability of multiple signals. This variability is not random but related to human, organizational and technical behaviors. The functional resonance is a dynamic phenomenon, depending on system and environmental conditions.

Based on these principles, safety assessment should go beyond traditional measures relying on the number of failures: things that go wrong (accidents and incidents) and things that go right (normal work) both arise from performance variability. Determining the safety level of a system consists thus of measuring the presence of acceptable outcomes: (obviously) the more, the safer. For risk assessment purpose, the FRAM can help identifying proper leading indicators for system safety (e.g. in the aviation domain ${ }^{21}$ : airworthy in terms of maintenance performance and vibration health monitoring; quality of communication; procedures quality by audits and observations, etc.). This perspective is in line with the principle of approximate adjustments, in the sense that a system can be advocated as robustly safe if it has the capability of adjusting its functioning to deal with both expected and unexpected working conditions. On the contrary, a system failure is the result of an unintended 
interaction of multiple signals, arising from normal performance variability and leading to disproportionately large effects arising from small or even insignificant variations.

The next sections $(\S 3.1$ - 3.4) summarize the FRAM’s four fundamental building steps, taking into account the innovative formulation of RAM, for the purpose of accident analysis.

\subsection{Step 1: Identification and description of system's functions}

Based on system knowledge and through the analysis of processes and tasks, available procedures, historic data, and/or involving SMEs, it is possible to characterize the system by its functional structure. In the FRAM, a function is made up of six aspects:

- Input (I): what starts the function or what is processed or transformed by the function.

- Output (0): the result of the function, either an entity or a state change, used as input to the downstream functions.

- Precondition (P): mandatory conditions that must exist before carrying out the function, not necessarily implying the function execution.

- $\quad$ Resource (R): what the function needs when it is carried out or consumes to produce the output.

- Control (C): function monitoring and controlling, regulating its performance to match the desired Output.

- Time (T): temporal constraints affecting the function, with regard to both duration and time of execution.

\subsection{Step 2: Identification of performance variability}

Since performance variability has a crucial role in everyday work, it is necessary to identify how each system function varies. Differently from risk assessment, in an accident analysis it is possible to define the actual variability during the accident itself. In this case, it is possible to define the variability in terms of phenotypes of variability, (e.g.) timing, precision, direction, speed, object, force ${ }^{1}$, with specific identification of the variability state. This variability represents a key to understand systematically the deviations from normal work and by the aid of FRAM Step 3 (see § 3.3), the propagation of these deviations within the system.

\subsection{Step 3: Aggregation of variability - the role of RAM}

This step aims to develop an overall description of the actual links and couplings among functions, related to the accident. This description highlights how variability propagates through the system, generating functional resonance or damping. Currently, the analysis requires the analyst to deal with variability, link by link, following the visual representation of FRAM functions. However, as discussed in recent literature for a risk assessment scenario, this standard analysis has some limitations in terms of comprehensibility of a complex scenario, which 
generates a complicated FRAM model, visually overloaded, i.e. including a great number of highly interconnected functions ${ }^{27}$.

In terms of aggregation of variability, the RAM presented in this paper, serves as a support tool to help visualizing functional interdependencies. A functional interdependence is a relationship that exists between two aspects so that an upstream aspect influences a different downstream aspect in a specific way, i.e. as Input, Precondition, Control, Resource or Time. Defining the functional interdependencies in a work domain is not an algorithmic process but rather it is a gradual development of the understanding process of the specific event under analysis, because of mutual understanding among the SMEs and the analysts involved in the analysis.

The matrix is constructed listing on the rows and on the columns the $n$ couplings of the model; therefore it is an $n$ $x n$ square matrix. By the term "coupling", we intend each type of output-input link, i.e. not only O-I but also O-P, O-C, O-R, O-T. As a difference from a previously presented matrix based on FRAM 5 , note that here we list the couplings rather than the functions. This choice represents a solution to highlight the relevance of studying components interactions rather than individual functions: the RAM we proposed relates couplings, with no need to detail the description of the aspects in each matrix' element, but rather allowing a more readable analysis in line with the principles of Safety-II. The element $R A M_{i j}$ assumes values 1 or 0 : if the coupling $i$ is linked through upstream functions to the coupling $j R A M_{i j}=1$, if not, $R A M_{i j}=0$. In case $R A M_{i j}=1$, the element also will bring the so-called (in IT language) attribute. The attribute generally defines a property of the element, i.e. the relationships between two functions, based on the nature of the link (I, P, C, R and T). Figure 1 (A) shows an example of a simple FRAM model with 6 functions and 8 couplings. Figure 1 (B) shows the respective RAM, highlighting the elements that have a connection in a systematic and well-organized way. Note that in this example each output is defined as a number $(1,2,3,4,5,6,7$ and 8), as detailed in Figure 1 (A). 


\section{A}

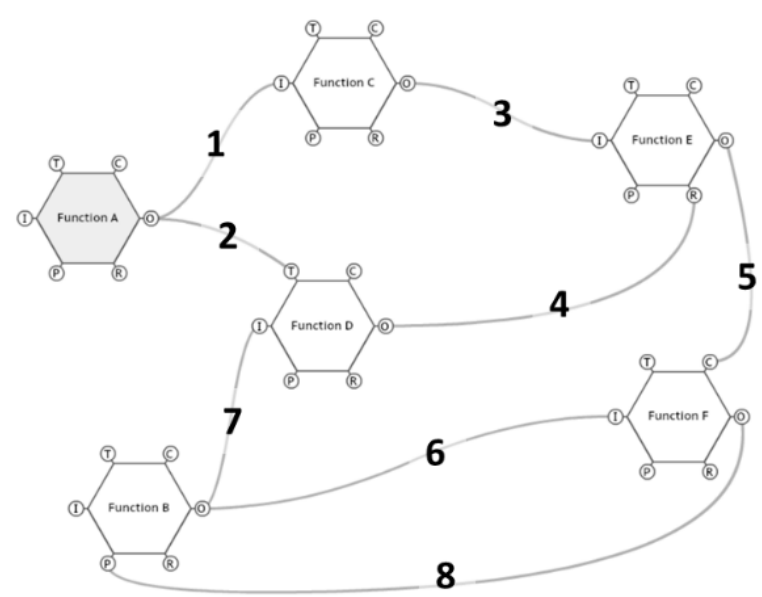

B

\begin{tabular}{|c|c|c|c|c|c|c|c|c|}
\hline & 1 & 2 & 3 & 4 & 5 & 6 & 7 & 8 \\
\hline 1 & 0 & 0 & 0 & 0 & 0 & 0 & 0 & 0 \\
\hline 2 & 0 & 0 & 0 & 0 & 0 & 0 & 0 & 0 \\
\hline 3 & $1(1)$ & 0 & 0 & 0 & 0 & 0 & 0 & 0 \\
\hline 4 & 0 & $1(\mathrm{~T})$ & 0 & 0 & 0 & 0 & $1(1)$ & 0 \\
\hline 5 & 0 & 0 & $1(\mathrm{I})$ & $1(\mathrm{R})$ & 0 & 0 & 0 & 0 \\
\hline 6 & 0 & 0 & 0 & 0 & 0 & 0 & 0 & $1(\mathrm{P})$ \\
\hline 7 & 0 & 0 & 0 & 0 & 0 & 0 & 0 & $1(\mathrm{P})$ \\
\hline 8 & 0 & 0 & 0 & 0 & $1(\mathrm{C})$ & $1(\mathrm{I})$ & 0 & 0 \\
\hline
\end{tabular}

Figure 1. A conceptual example of RAM applied to FRAM.

A connection between two or more couplings highlights a potential source of functional resonance in case the upstream coupling is subjected to a not-negligible internal or external variability. Therefore, the RAM allows discussing the effects of propagation of variability to downstream functions or its upstream origin, taking advantage of a systematic representation of the variability paths.

For the purpose of an accident analysis, it would be of interest to investigate how a specific coupling affects the system (looking at its downstream couplings) or is affected by the system functioning, i.e. by the upstream couplings. For example, in case it would be necessary to look at how a relevant variability of the couplings $j$ affects the system, there could be useful to follow this simple algorithm:
A. pick the $j$-th coupling and mark the corresponding element in the heading row of the RAM
B. $\operatorname{assign} i_{k 2}=j$ and $i_{k 1}=1$
C. check if any element $R A M_{i_{k 1} i_{k 2}}=1$ (for $i_{k 2}=j$ and $i_{k 1}=1 \ldots, n$ ) and mark them
D. when condition at point $\mathrm{C}$ is verified, assign $i_{k 2}=i_{k 1}$ and $i_{k 1}=1$
E. iterate step $C$ until one of these two conditions is verified: there is no $R A M_{i_{k 1} i_{k 2}}=1$; or if $\left(i_{k 2}, i_{k 1}\right)$ acquires yet assigned values (i.e. there is a closed loop).

Figure 2 shows an example of the application of the algorithm to evaluate how the inherent variability of coupling 7 affects other couplings in the model. It highlights respectively the following links: I-O (7 and 4), R-O (4 and 5), C$\mathrm{O}$ (5 and 8), P-O (8 and 6), I-O (6 and 8) and P-O (8 and 7), before verifying a stop condition, i.e. entering in a closed loop. Similar observations can be defined to understand the upstream sources of variability, simply adapting this reasoning. 


\begin{tabular}{|c|c|c|c|c|c|c|c|}
\hline & 1 & 2 & 3 & 4 & 5 & 6 & 8 \\
\hline 1 & 0 & 0 & 0 & 0 & 0 & 0 & 0 \\
\hline 2 & 0 & 0 & 0 & 0 & 0 & 0 & 0 \\
\hline 3 & 1 (I) & 0 & 0 & 0 & 0 & 0 & 0 \\
\hline 4 & 0 & $1(\mathrm{~T})$ & 0 & & & & 0 \\
\hline 5 & 0 & 0 & 1 (I) & 1 (R) & & 0 & 0 \\
\hline 6 & 0 & 0 & 0 & 0 & & & \\
\hline 7 & 0 & 0 & 0 & 0 & & & \\
\hline 8 & 0 & 0 & 0 & 0 & & & \\
\hline
\end{tabular}

Figure 2. An analysis of downstream couplings in a conceptual RAM applied to FRAM.

Furthermore, it is possible to take advantage of the attribute each $R A M_{i j}=1$ is carrying $(\mathrm{I}, \mathrm{P}, \mathrm{C}, \mathrm{T}, \mathrm{R})$. This attribute would guide the analysis through the path, to translate the FRAM hexagons into a logic representation of each connection. This analysis requires understanding the upstream-downstream coupling, looking in further detail at how differences in the quality of upstream output can affect the variability of downstream functions. For example, an upstream Output representing a Precondition of a downstream function, would be critical if too early, causing a potential false start, or even more critical if omitted, requiring improvisation, in both cases amplifying the effects of variability. Therefore, a medium level of variability of the Output may become high for the coupling under analysis (O-P). The same Output, if is a Resource for a different downstream function, might have a less critical even positive - effect on the coupling: the Output' medium level in terms of variability may become a low level of variability for the coupling (O-R). Starting from the conceptual taxonomy of a generic socio-technical system, see Chapter 7 of Hollnagel's book on FRAM ${ }^{1}$, it is possible to describe each link by its own, using the combined knowledge of the SMEs involved in the analysis to understand if the Output's variability is damped or amplified when connected to another aspect.

Table 1 summarizes these observations by the SMEs involved in the analysis, showing how the inherent variability of an upstream Output may affect the interconnected functions: it amplifies, damps or has no effect, depending on the connection upstream/downstream connection. 
Table 1. Upstream/Downstream propagation of variability

\begin{tabular}{|c|c|c|c|c|c|c|}
\hline & & Input & Precondition & Resource & Control & Time \\
\hline \multirow{4}{*}{$\begin{array}{l}\text { Timing } \\
\text { Variability } \\
\text { of Output }\end{array}$} & Too Early & $\begin{array}{l}\text { Amplifying/ } \\
\text { No Effect }\end{array}$ & Amplifying & $\begin{array}{l}\text { No Effect/ } \\
\text { Damping }\end{array}$ & Amplifying & Amplifying \\
\hline & On time & Damping & Damping & Damping & Damping & Damping \\
\hline & Too Late & Amplifying & Amplifying & Amplifying & Amplifying & Amplifying \\
\hline & Not at all & Amplifying & Amplifying & Amplifying & Amplifying & Amplifying \\
\hline \multirow{3}{*}{$\begin{array}{l}\text { Precision } \\
\text { Variability } \\
\text { of Output }\end{array}$} & Imprecise & Amplifying & Amplifying & Amplifying & Amplifying & Amplifying \\
\hline & Acceptable & No Effect & No Effect & No Effect & No Effect & No Effect \\
\hline & Precise & Damping & Damping & Damping & Damping & Damping \\
\hline
\end{tabular}

In addition, there would be possible to develop a graphical representation of the couplings in the RAM, by a colorcoded variability scale (from red to green, see for example Figure 3).

\begin{tabular}{|c|c|c|c|c|c|c|c|c|}
\hline & 1 & 2 & 3 & 4 & 5 & 6 & 7 & 8 \\
\hline 1 & 0 & 0 & 0 & 0 & 0 & 0 & 0 & 0 \\
\hline 2 & 0 & 0 & 0 & 0 & 0 & 0 & 0 & 0 \\
\hline 3 & 1 (I) & 0 & 0 & 0 & 0 & 0 & 0 & 0 \\
\hline 4 & 0 & $1(\mathrm{~T})$ & 0 & 0 & 0 & 0 & 1 (I) & 0 \\
\hline 5 & 0 & 0 & 1 (I) & 1 (R) & 0 & 0 & 0 & 0 \\
\hline 6 & 0 & 0 & 0 & 0 & 0 & 0 & 0 & $1(\mathrm{P})$ \\
\hline 7 & 0 & 0 & 0 & 0 & 0 & 0 & 0 & $1(\mathrm{P})$ \\
\hline 8 & 0 & 0 & 0 & 0 & 1 (C) & 1 (I) & 0 & 0 \\
\hline
\end{tabular}

Figure 3. A conceptual analysis of variability links in a RAM applied to a simple FRAM model.

This assessment can be linked to Perrow's Normal Accident Theory (NAT): the essence of accidents in complex systems is the interaction of multiple unavoidable failures that are not in a direct operation sequence. Perrow links the concept of system safety to the number of interconnections of an item ${ }^{28}$. Consequently, it would be possible to create indexes based on the number of interconnections, as a means to identify highly connected couplings and then potential criticalities. This perspective reflects the idea that a coupling deriving from an upstream function, common to a group of couplings, might generate potential critical effects in the system, once that upstream output is released as variable. Based on RAM, we suggest two indexes, respectively considering the number of upstream links (UL) of a coupling and the downstream links (DL) of a $j$-th coupling: $U L_{j}=\sum_{w}^{n} R A M_{j w}$ and $D L_{j}=\sum_{w}^{n} R A M_{w j}$. 
The higher the values of UL and DL and the more interconnected is the coupling (upstream or downstream), and consequently the higher the potential to propagate functional resonance within the system, leading to emergent events. These observations, which might appear trivial in this simplified model, would acquire a crucial role in more interconnected models, where a simple visual discussion would not allow a complete and systematic analysis.

\subsection{Step 4: Management of variability}

The purpose of this step consists of understanding the complexity of the work domain. At this step, the simplest analysis consists of looking if there is any simple cause-effect link due to the excess of variability of specific functions, even arising as a combination of multiple sources of variability. Furthermore, the FRAM especially with the aid of RAM, acknowledges the dynamic non-linear essence of couplings, and focuses on finding the tight couplings and complex interactions that can explain the event. The final step of the investigation process is linked not only to the definition of barriers and to the detection of latent conditions. It also looks at how performance variability can be monitored and managed, specifically looking at how variability can be managed with no negative effects on system productivity, i.e. acknowledging that a portion of variability in everyday work in unavoidable to ensure both safety, productivity and quality. Therefore, a FRAM model might provide guidelines for defining performance indexes to monitor system's variability, because of a RAM-based systematic assessment.

\section{Illustrative case study}

On a world base, the majority of accidents involving Air Traffic Management (ATM) occurred during the ground phase of flight ${ }^{29}$. One of the most important safety events in the ground phase is the so-called runway incursion (RI-VAP), defined by ICAO as "any occurrence at an aerodrome involving the incorrect presence of an aircraft, vehicle, or person on the protected area of a surface designated for the landing and take-off of aircraft" ${ }^{30}$. A RI-VAP can be categorized using different grade of severity, ranging from D to A, where the most severe ones (grade A - B) include a near collision and a collision. ATCOs have a crucial role for preventing potential RI-VAP, which usually represents an emergent event rather than a simple cause-effect relationship. On this path, to deal with RI-VAP, it becomes necessary to be proactive rather than reactive to manage variability's propagation, when necessary. It becomes necessary thus to act in terms of Safety-II, looking at system functioning and at the inter-relations among the system's agents rather than the single probability of failure. In terms of communications among pilot and ATCOs, (e.g.) a failure can be a misunderstanding: a disagreement, a difference of opinion, or more specifically a failure to understand or interpret correctly someone or something 30,31 . 
This section describes the development of a FRAM model to analyse in-depth the system functioning, in a scenario leading to a runway incursion in LAX airport (1991). The model would allow the analysis of functions performed by ATCOs, in charge of responsibility for moving the aircraft from the apron to the holding point of the assigned runway, by pilots and by airport personnel, working for the ground-handling provider, in charge of inspect the runway. Each agent is also discussed looking at the organizational aspects related to his actions. The analysis is focused on the departure of an aircraft, on how the required functions to realize the operations in a well safe, efficient, effective and operational scenario, interact with each other.

Note that this illustrative case study does not aim to develop a new complete accident analysis, but rather to show the potential of FRAM and RAM, in a complex work domain.

\subsection{Description of the event}

On January, $1^{\text {st }}$ 1991, ATC system at LAX (Los Angeles International airport) was classified as level V (the one allowing the maximum number of movements at that time) ${ }^{32}$. This classification was used to define an ATC tower able to manage more than 100 movements per hour, 16 hours per day, with 632.312 total movements in 1990 . The accident happened one hour after the sunset. In the tower, there were 13 ATCOs. The Local Controller 2 (LC2) was the ATCO in charge of the RWY 24L, when the event occurred. LC2 used frequency $133.900 \mathrm{MHz}$ and was engaged in a routinely work shift: during night, with many aircrafts landing and departing every 50 seconds, using 2 RWYs at the same time for both landing and departing activity, with a consequently complex taxiway net. Frequency was extremely busy, due to the numerous communications from and for pilots. With two aircraft at the holding point of RWY 24L, with others 15 engaging the frequency, flight SKW5569 (SkyWest), taxing on the maneuvering area, asked to depart from intermediate intersection 45 . The LC2 did authorize the intermediate line up, while busy in re-establishing communications with WW5006 (West Wings), which was authorized to cross RWY 24L, close to the end of runway. There were numerous communications with that aircraft, since it erroneously changed frequency. At the same time, the approaching flight USair 1493, after not being able to established radio communications with the TWR, reiterate its presence at 18:04:35, by then at the very short final. Having already done all the controls only missed the landing clearance. Among the first radio contact of USair1493 and its second communication, in 1 minute and 30 seconds, the LC2 gave and received 40 communications. Into this temporal interval, SKW5569 asked for lining up using intermediate intersection 45 . The LC2 gave the authorization while USair1493 was approaching the threshold 24: it was authorized to land at 18:05:51. Among the second communication of USair1493 and its landing clearance, the LC2 gave and received 22 others calls. In 
the meantime, SKW5569 have been waiting on the RWY 24L for 2 minutes and 17 seconds. The impact occurred at 18:06:59, as deducted from VCR of the USAir1493.

The NTSB's conclusion defines the LC2's role in the event as a metaphoric iceberg's peak, whose mass was, as a whole, the actual messy reality behind the event. Therefore, in a complex socio-technical system, the analysis of action has to be contextualized in the specific operational situation itself, in order to understand the local rationality of the specific event. This local rationality plays a crucial role to understand the ATCO's actions and behaviours. Considering his/her role as a sharp end operator, his/her actions relies on all the operators' working actions and operating conditions. This central role becomes evident in the event of USair1493 accident, requiring a systemic perspective to allow a reliable accident analysis. FRAM would help performing this analysis, as detailed in the next steps.

\subsection{FRAM Step 1 for the USAir 1943 accident}

Starting from the NTSB report, testimonies by people involved in the event, documents and coeval accounts, and moreover based on three SMEs' analysis of the event, the FRAM model discussed in this paper counts 57 functions (organizational, technological and human) interconnected with each other by $n=245$ couplings. Since some of these functions can be considered as collectors of performance variability, it would be even possible to develop a more detailed model, to address specific features of the accident. These numbers based on a real scenario reflects a complicated model. Table 2 lists the functions involved in the analysis, detailing the agent performing them.

Table 2. List of functions of the FRAM model

\begin{tabular}{|l|l|}
\hline Function & Agent \\
\hline Give a take-off Clearance & ATCO \\
\hline Taxi & Pilot \\
\hline Give a taxi clearance & ATCO \\
\hline Request a taxi clearance & Pilot \\
\hline Take weather and airport data (Notams) & Pilot \\
\hline Make a taxi briefing & Pilot \\
\hline Perform a taxi checklist & Pilot \\
\hline Request clearance(s) from ATC & Pilot \\
\hline Take off briefing & Pilot \\
\hline Perform a before take-off checklist & Pilot \\
\hline Request a clearance for take off & Pilot \\
\hline Line up on the runway & Pilot \\
\hline Prepare flight info (by flight dispatcher) & Organization/Pilot \\
\hline Give weather and airport data & ATCO \\
\hline Request start up and push back & Pilot \\
\hline Give clearance for start-up and push back & ATCO \\
\hline Observe weather phenomena & Organization/ATCO \\
\hline Coordinate with APP & Organization/ATCO \\
\hline Coordinate with ACC & Organization/ATCO \\
\hline Meteo data & Technology/ATCO \\
\hline Organise operations strategically & Organization/ATCO \\
\hline Check inbound-outbound list & ATCO \\
\hline Experience & Organization/ATCO \\
\hline Assess A/C position from working position & ATCO \\
\hline & \\
\hline
\end{tabular}




\begin{tabular}{|c|c|}
\hline Ambient working condition & Organization/ATCO \\
\hline Cockpit working condition & Organization/Pilot \\
\hline TFM & Organization/ATCO \\
\hline HMI & Organization/ATCO \\
\hline Give ATC clearance & ATCO \\
\hline Be operative & ATCO \\
\hline Engage the RWY & Pilot \\
\hline FMS & Technology/Pilot \\
\hline Manage the FMS & Pilot \\
\hline Handler & Organization/Airport facility \\
\hline Readback & ATCO \\
\hline Hearback & ATCO \\
\hline Sterile cockpit & Organization /Pilot \\
\hline SGS working & Technology/ATCO \\
\hline ASDE working & Technology/ATCO \\
\hline Follow the surface markings & Pilot \\
\hline Entry procedures complied & ATCO \\
\hline TWYs system & Organization/ATCO \\
\hline Give an intermediate line up clearance & ATCO \\
\hline Request clearance for intermediate take off & Pilot \\
\hline Organise operations tactically & Organization/ATCO \\
\hline Manage flight strips & ATCO \\
\hline Monitor frequency & Pilot \\
\hline Communication style & ATCO \\
\hline Radio apparatus working & Technology/ATCO \\
\hline BRITE working & Technology/ATCO \\
\hline Evaluate ATC features & Organization/ATCO \\
\hline Radio communication phraseology and techniques & Organization/ATCO \\
\hline Manage air traffic volume & Organization/ATCO \\
\hline Coordinate movements & ATCO \\
\hline Request landing clearance & Pilot \\
\hline Give landing clearance & ATCO \\
\hline Maintain RWY holding point & Pilot \\
\hline
\end{tabular}

\subsection{FRAM Step 2 for the USAir 1943 accident}

Firstly, functions have been characterized individually, describing all the necessary aspects and phenotypes of their actual variability during the event, which has been described by the SMEs involved in the analysis, following Hollnagel's simple solution, i.e. in terms of timing and precision ${ }^{1}$. Subsequently, the origin of the variability was identified for all the Outputs, as the result of both internal and external variability, besides upstream-downstream couplings. The SMEs assign also a rank to variability for each functions, based on their experience on the field with the knowledge obtained about the specific event, by a simple color-coded scale on three values (see Table 3):

- Red, function's timing and precision have a serious effect on how downstream functions are performed

- Yellow, function's timing and precision have a potential effect on how downstream functions are performed

- Green, functions' timing and precision have a limited or even negligible effect on the system, with no consequences on how downstream functions are performed. 
Table 3. Variability values for each function and criticality scale

\begin{tabular}{|c|c|c|c|}
\hline Function & Timing & Precision & Criticality \\
\hline Give a take-off Clearance & Not at all & Imprecise & \\
\hline Taxi & Too late & Imprecise & \\
\hline Give a taxi clearance & On time & Precise & \\
\hline Request a taxi clearance & On time & Precise & \\
\hline Take weather and airport data (Notams) & On time & Precise & \\
\hline Make a taxi briefing & On time & Imprecise & \\
\hline Perform a taxi checklist & On time & Precise & \\
\hline Request clearance(s) from ATC & On time & Precise & \\
\hline Take off briefing & On time & Imprecise & \\
\hline Perform a before take-off checklist & Too early & Precise & \\
\hline Request a clearance for take off & On time & Precise & \\
\hline Line up on the runway & On time & Imprecise & \\
\hline Prepare flight info (by flight dispatcher) & On time & Precise & \\
\hline Give weather and airport data & On time & Precise & \\
\hline Request start up and push back & On time & Precise & \\
\hline Give clearance for start-up and push back & On time & Imprecise & \\
\hline Observe weather phenomena & On time & Precise & \\
\hline Coordinate with APP & On time & Acceptable & \\
\hline Coordinate with ACC & On time & Precise & \\
\hline Meteo data & On time & Precise & \\
\hline Organise operations strategically & Not at all & Imprecise & \\
\hline Check inbound-outbound list & On time & Precise & \\
\hline Experience & Not at all & Imprecise & \\
\hline Assess A/C position from working position & Not at all & Imprecise & \\
\hline Ambient working condition & Not at all & Imprecise & \\
\hline Cockpit working condition & On time & Precise & \\
\hline TFM & On time & Precise & \\
\hline HMI & Not at all & Imprecise & \\
\hline Give ATC clearance & On time & Precise & \\
\hline Be operative & On time & Precise & \\
\hline Engage the RWY & Too early & Imprecise & \\
\hline FMS & On time & Precise & \\
\hline Manage the FMS & On time & Precise & \\
\hline Handler & On time & Precise & \\
\hline Readback & Not at all & Imprecise & \\
\hline Hearback & Not at all & Imprecise & \\
\hline Steryle cockpit & On time & Precise & \\
\hline SGS working & On time & Precise & \\
\hline ASDE working & Not at all & Imprecise & \\
\hline Follow the surface markings & On time & Precise & \\
\hline Entry procedures & Too early & Imprecise & \\
\hline TWYs system working & On time & Precise & \\
\hline Give an intermediate line up clearance & Too early & Imprecise & \\
\hline Request clearance for intermediate take off & On time & Imprecise & \\
\hline Organise operations tactically & Not at all & Imprecise & \\
\hline Manage flight strips & Not at al & Imprecise & \\
\hline Monitor frequency & Not at all & Imprecise & \\
\hline Communication style & On time & Imprecise & \\
\hline Radio apparatus working & On time & Precise & \\
\hline BRITE working & On time & Imprecise & \\
\hline Evaluate ATC features & On time & Imprecise & \\
\hline Radio communication phraseology and techniques & Too late & Imprecise & \\
\hline Manage air traffic volume & Not at all & Imprecise & \\
\hline Coordinate movements & Not at all & Imprecise & \\
\hline Request landing clearance & On time & Acceptable & \\
\hline Give landing clearance & Too early & Imprecise & \\
\hline Maintain RWY holding point & On time & Precise & \\
\hline
\end{tabular}

\subsection{FRAM Step 3 for the USAir 1943 accident}

Once identified the variability of each function, it is necessary to evaluate each coupling with other functions.

Figure 4 depicts the overall FRAM model, which offers an overview of the inherent complexity of the process, and the subsequent inherent issues related to its analysis. In this case, as well as in many practical application of the method, the traditional analysis based on the visual representation of the model might offers limited help. For this 
purpose, the RAM would help highlighting all the couplings relations, and thus helping identifying those potential paths where the functional resonance might propagate. Figure 5 depicts the color-coded RAM, based on the assessment included in Table 3 and the SMEs' critical observation to define the effects of variability in downstream and upstream couplings, based on the nature of the link and of the function (see Chapter 7 of Hollnagel's FRAM book $^{1}$ ). Figure 6 shows the complete RAM, where the elements $R A M_{i j}=1$ are characterized by a blue background color. Figure 6 shows also each one of the 245 couplings, in terms of its UL and DL, coding their value in a colorcoded scale respectively in the last column (UL) and row (DL). The scale (green, yellow, red) is directly proportional to UL and DL values, whose range in this case are respectively $(0,24)$ and $(0,21)$. For more details, Figure 7 details the occurrences of couplings in the same color-coded scale, specifying the focus on initial analysis, as discussed in $§ 4.5$. At this step, analyzing in detail the work-as-done through the RAM, it is possible to understand how the functional resonance propagated throughout the system, applying and iterating the procedure described in $\S 3.3$. 

https://doi.org/10.1142/S0218539318500018

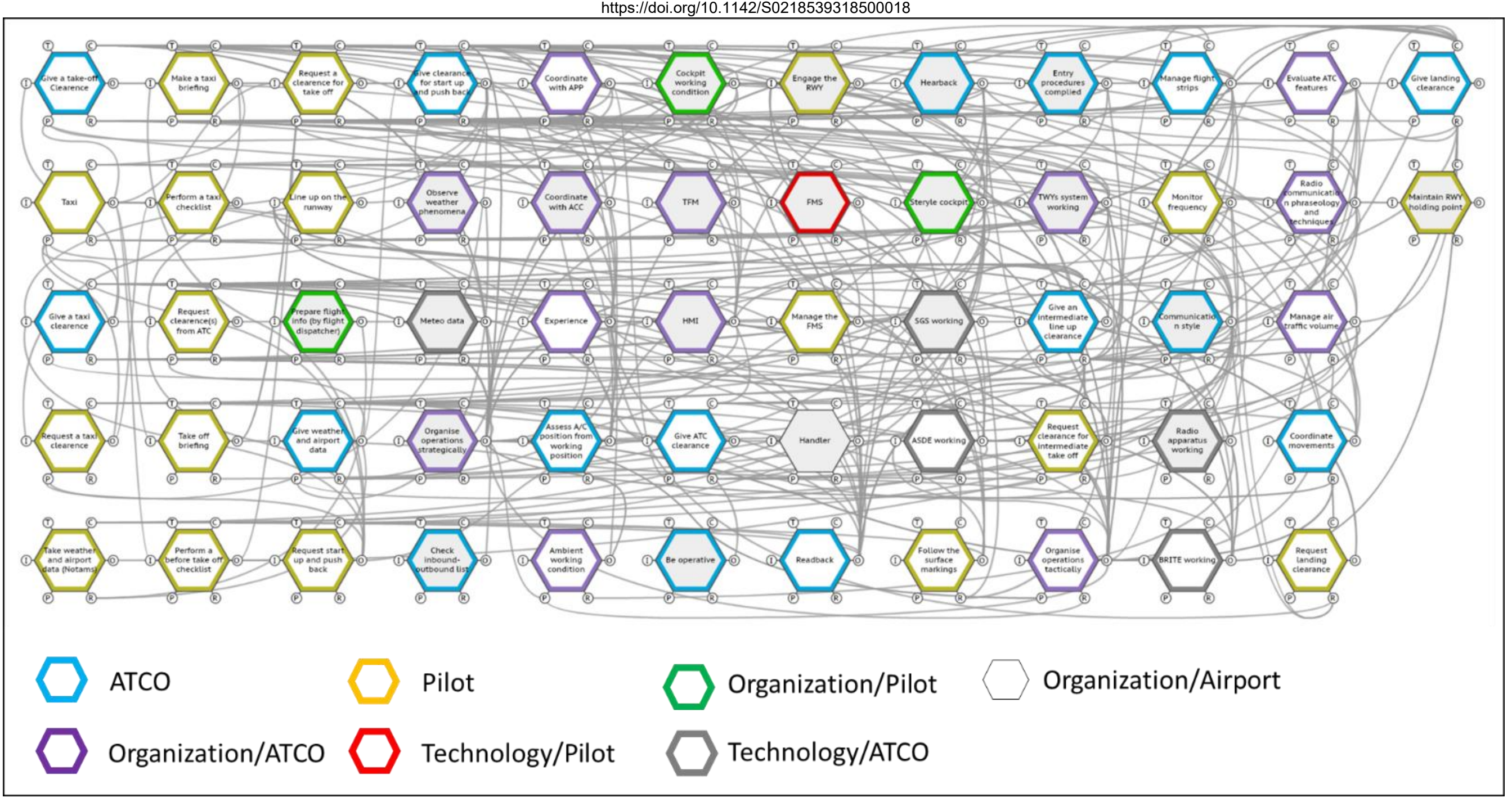

Figure 4. The complete FRAM model for the USair1943 accident. 
International Journal of Reliability, Quality and Safety Engineering

Patriarca Riccardo, Del Pinto Gianluca, Di Gravio Giulio, and Costantino Francesco

https://doi.org/10.1142/S0218539318500018

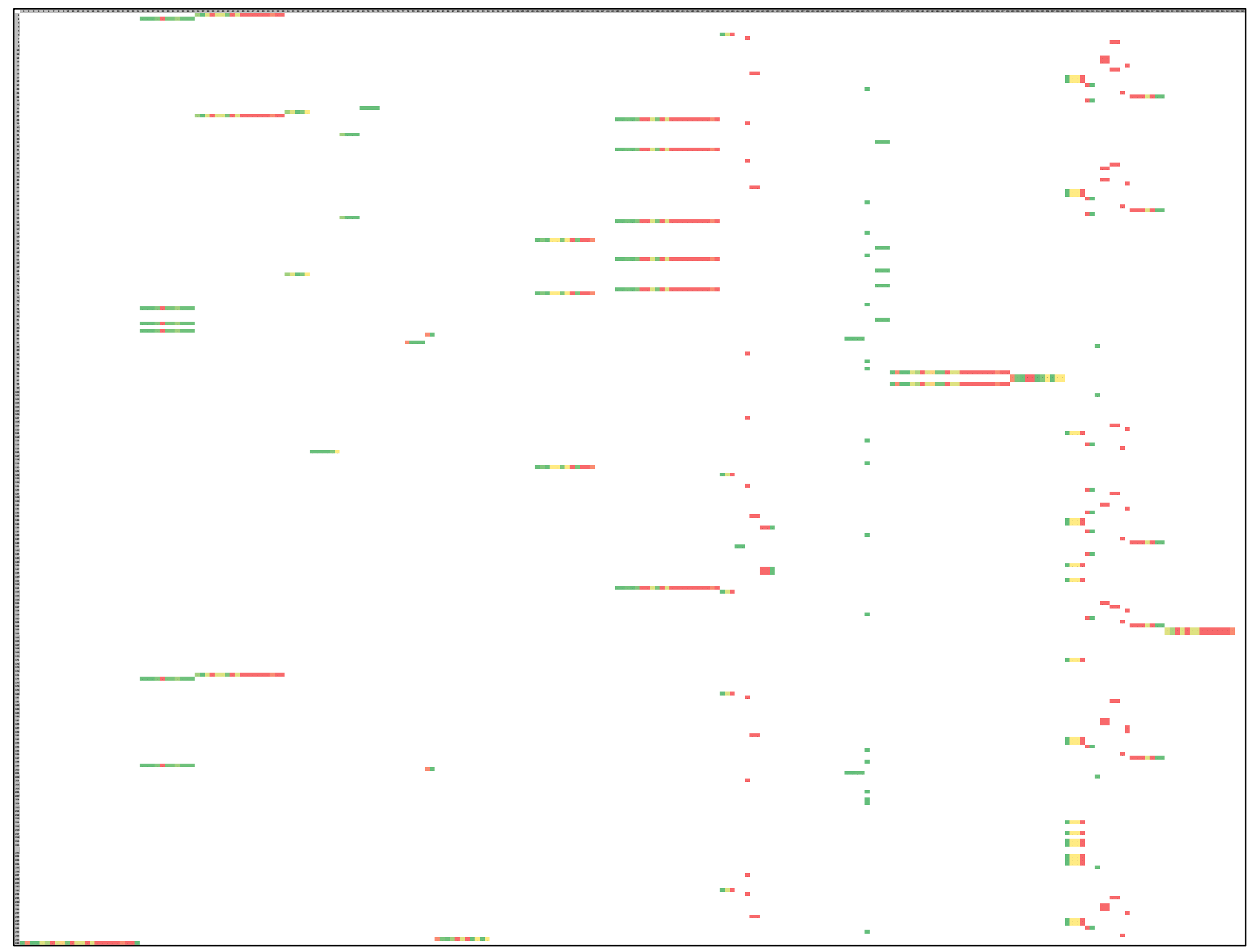

Figure 5. The color-coded RAM based on the FRAM model for the USair1943 accident. 
International Journal of Reliability, Quality and Safety Engineering

Patriarca Riccardo, Del Pinto Gianluca, Di Gravio Giulio, and Costantino Francesco

https://doi.org/10.1142/S0218539318500018

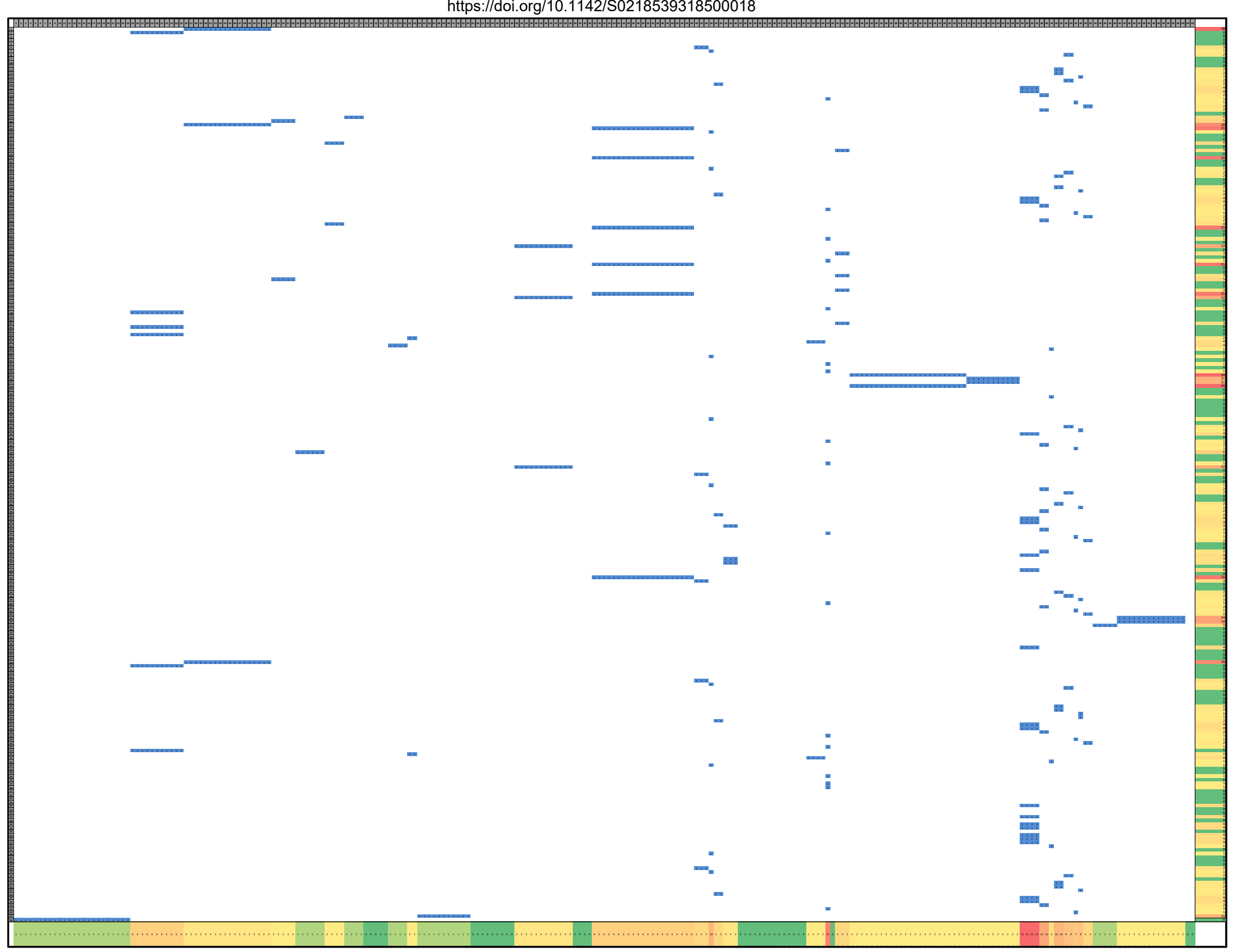

Figure 6. The complete FRAM model for the USair1943 accident, showing UL and DL for each coupling. 


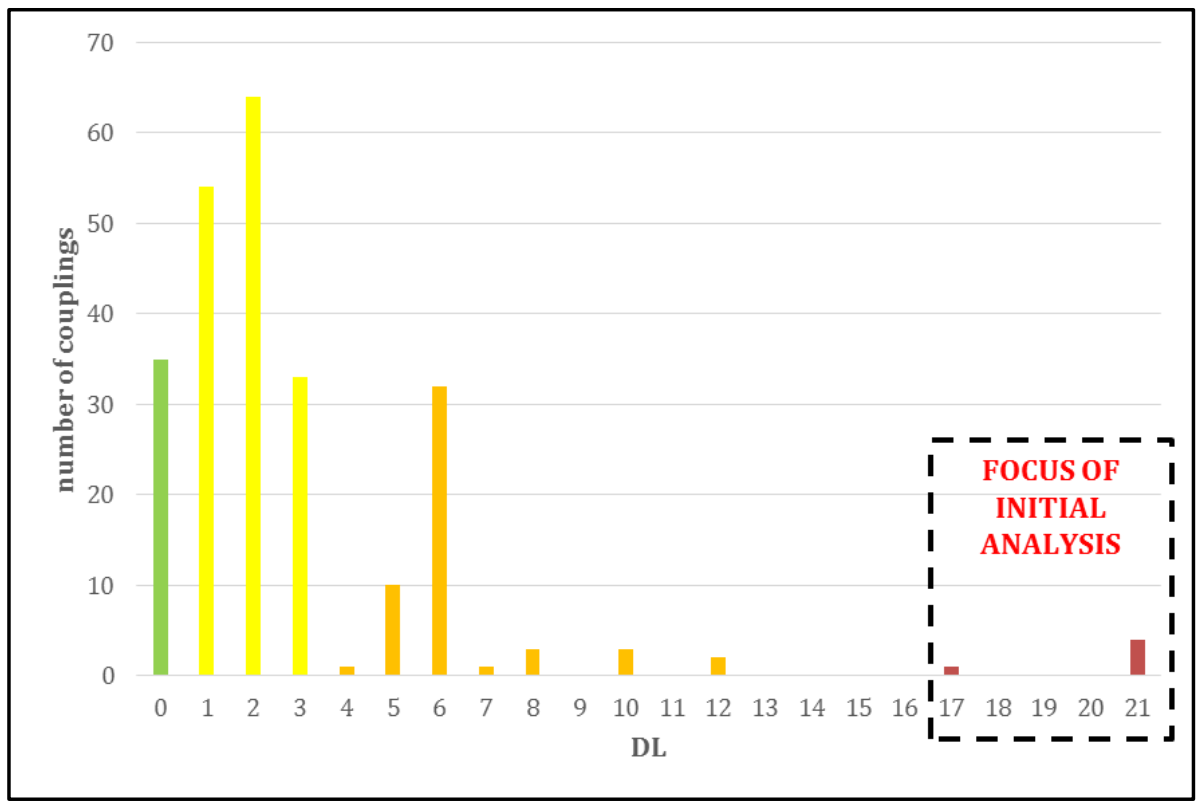

Figure 7. Formal representation of DL (as emerged from the RAM) and focus of initial analysis on the highest values of $D L$

\subsection{FRAM Step 4 for the USAir 1943 accident}

Lastly, for the purpose of step 4, once extrapolated the downstream and upstream couplings, it is necessary to focus on how non-linear interactions might affect the system variability, by the aid of the RAM, rather than the FRAM visual model. For example, we use the RAM to focus on the downstream and upstream couplings of functions characterized by a potentially serious variability in the scenario, i.e. $<$ Readback $>$ and $<$ Hearback> (both "Not at all" and "Imprecise", see Table 3), and their relationships with an organizational function, i.e. < Organize operations tactically> which also is critical in terms of variability ("Not at all" and "imprecise", see Table 3). Conceptually, the FRAM analysis about < Readback $>$ and similarly $<$ Hearback $>$, confirms that these functions act as intermediary between the organizational functions and the operational ones. However, it is difficult to identify and represent this correlation by traditional Safety-I reasoning and methods. Traditional analyses generally focus at each one of these levels, developing independent safety assessments (or dependent via direct and static causal correlations).

More in detail, the couplings <National OPS applied-Readback> (coupling number 170 ) <National OPS applied-Hearback> (coupling number 172) are two of the most critical couplings in terms of downstream links (DL=17) with limited upstream influences (UL=4). Furthermore, they are extremely critical if considering the effects of <National OPS applied>, i.e. OPS actually not applied, acts as Input for both of them. In this case, both functions <Readback> and < Hearback> would not be carried out properly, amplifying the variability of the upstream Output generating potential loss of time, loss of accuracy and misunderstandings. 
Therefore, these observation lead to focus primarily on the downstream effects of their variability, and secondarily on the upstream couplings. Adopting the algorithm described in $§ 3.3$ and using the color-coded matrix shown in Figure 6, it would be possible to highlight those couplings with critical effects on the systems (red, or yellow in Table 3). An example of iteration of the downstream and upstream iteration of the algorithm, would allow relating functions at different abstraction levels (tactical, operational), as shown in Figure 8. Note that the sinusoids in the hexagons indicate the functions characterized by a not negligible criticality in terms of variability (red, or yellow in Table 3).

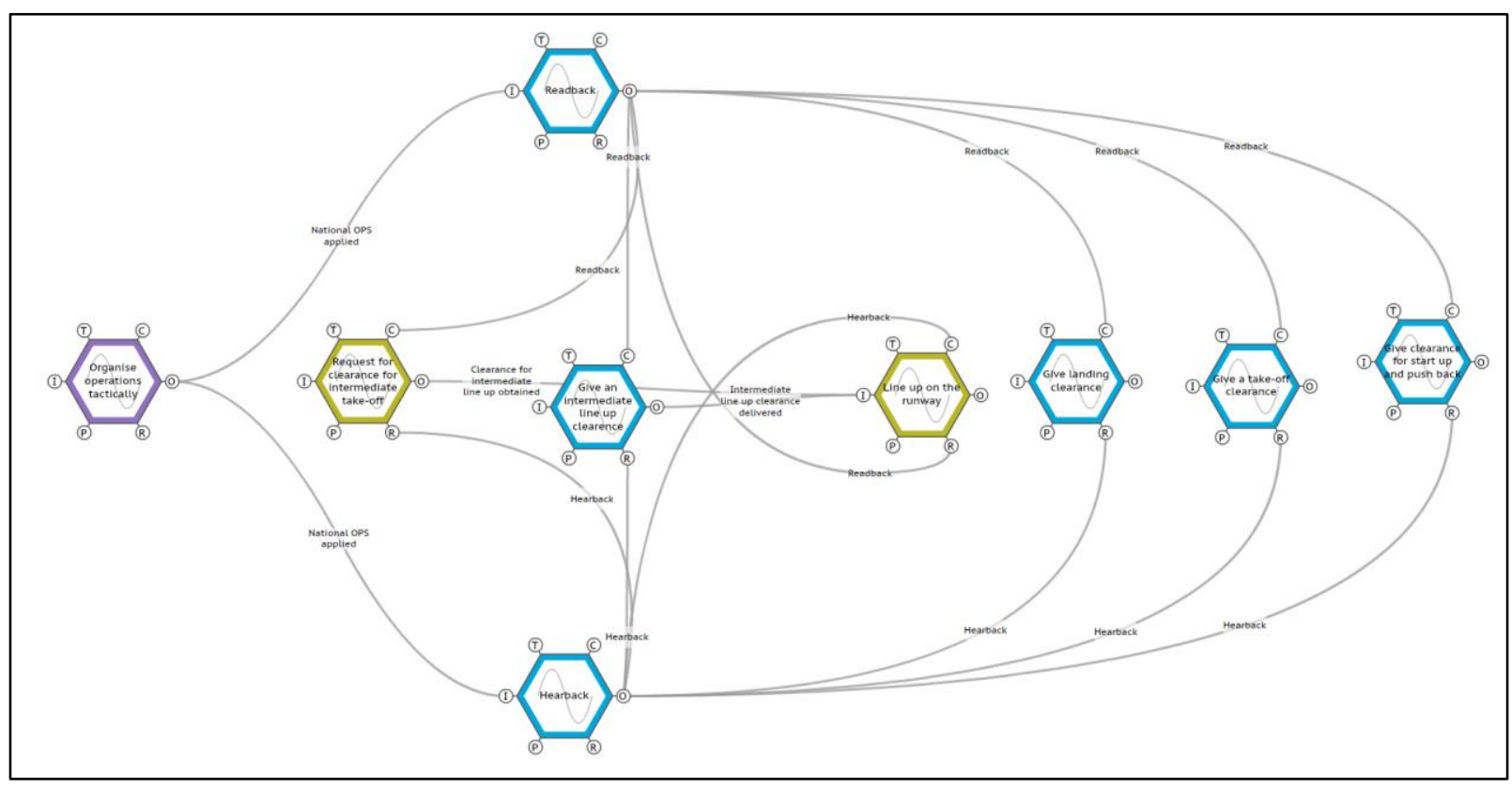

Figure 8. Example connections for $<$ Readback $>$ and $<$ Hearback $>$ with not-negligilble effect on the system (red or yellow couplings).

Furthermore, it is possible to use the RAM again, in order to identify completely the systemic effects, in terms of upstream and downstream couplings, significant in terms of variability, still adopting the colorcoded algorithm in Figure 5. More in detail, (e.g.) about $<$ Readback $>$, we started listening the radio communications related to the event, in order to define in detail how the variability propagates. We observed that $<$ Readback $>$ hardly ever respects those performance limits established at the organizational level. Moreover, $<$ Readback> being a human function, is inherently variable, and its variability allows the functioning of the system by the ATCOs who monitor continuously performance and take actions following expected or unexpected events.

On this context, it is relevant to look at the Airman's Information Manual (Chapter 2, section 4. Radio communication and phraseology and techniques -the edition in force-) which stated: Brevity is important, and contacts should be kept as brief as possible, but the controller must know exactly what you can do before he can properly carry out his control duties. And you, the pilot, must know exactly what he wants you. 
The Manual acknowledged brevity as crucial and demanded for contacts as short as possible: this reasoning implies the ATCO to be prepared, knowing exactly what to do before and during its work activities, and the pilot to be prepared to answer promptly the ATCO's requests. This aleatory statement might be properly identified and assessed, only when its variability is calculated and reported (e.g. in terms of timing and precision), reactively looking at the past events, or proactively to define safety measures.

For example, Figure 9 clarifies how < Readback $>$ supervises, as Control, the acceptability of most functions. It intervenes repeatedly, so that it represents a reference point from which an excess of performance variability can accumulate (because of its redundancy) and propagate through the operational scenario: note for example the two links with $<$ Request clearance for intermediate take off>, i.e. the two close communications at 18:03:38 and 18:03:44 (see Figure 9). The coupling between $<$ Readback $>$ and $<$ Request clearance for intermediate take off $>$ has a strong impact within the system $(\mathrm{DL}=21)$ and thus it has to be managed properly in terms of upstream variability, i.e. <Readback>. In the meantime, just to confirm the repetitiveness of $<$ Readback $>$, note the link with <Give an intermediate line up clearance $>$ at 18.03.40 (see Figure 9). This analysis confirms the crucial role of $<$ Readback $>$ function, to be considered as a potential source of amplification for the variability even among different agents, i.e. ATCO (blue hexagons) and pilot (yellow hexagons).

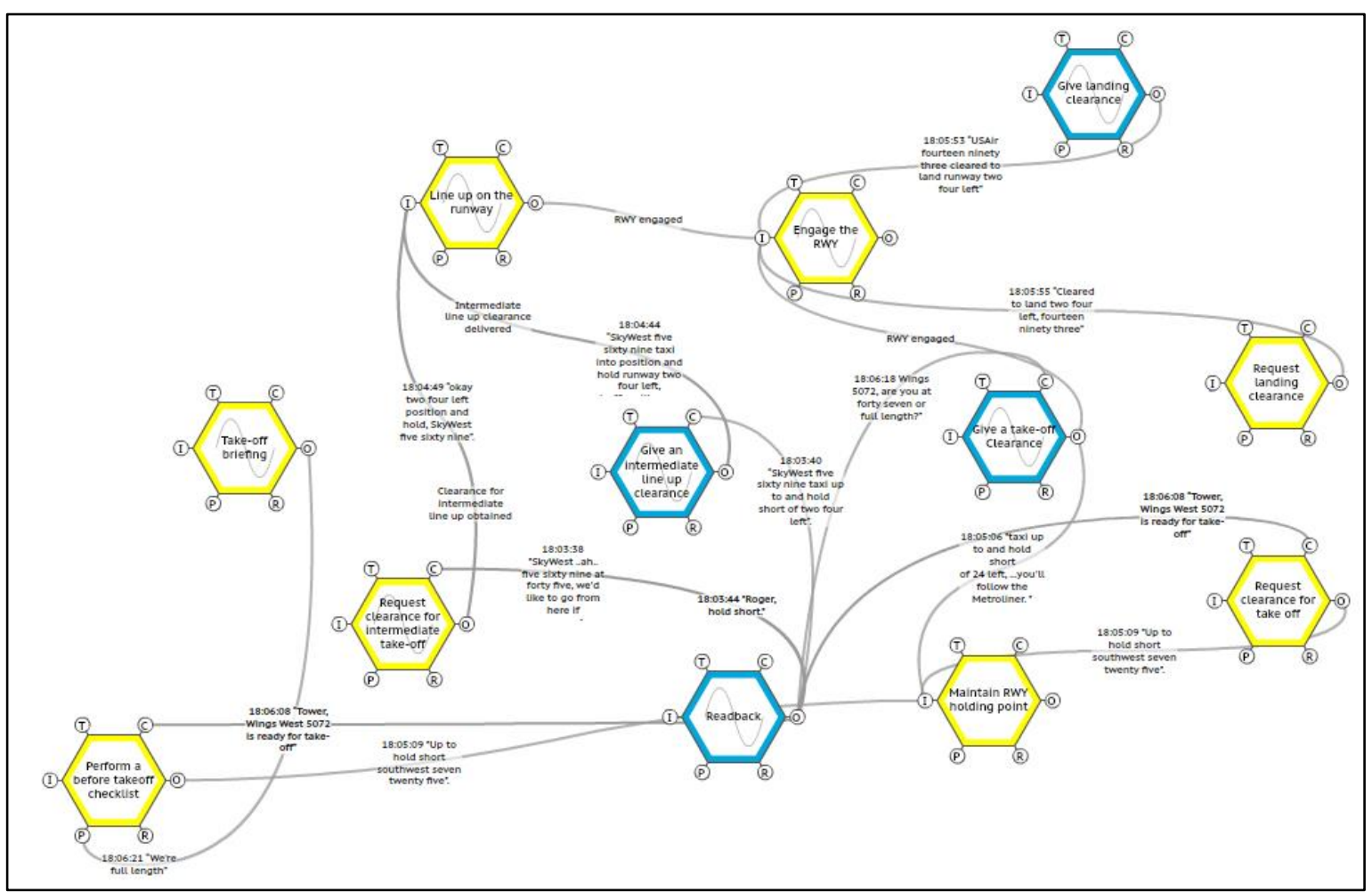

Figure 9. The most relevant (red or yellow couplings in terms of criticality) systemic effects of $<$ Readback $>$ in the USair 1943 accident. 
Another example of the relevance of the benefits of RAM would lead to track the couplings of a critically positioned function, i.e. <Manage flight strips>. Figure 10 shows the functional interdependencies among organizational functions at the blunt-end (the purple hexagons: <Organise operations tactically> and $<$ Organise operations strategically>), and operational functions at the sharp-end (the blue hexagons: <Give clearance for start-up and push back>, <Give a taxi clearance>, <Give an intermediate line up clearance $>$, $<$ Give a take-off clearance>, <Give landing clearance $>$ ). In particular, during the event, the Output of $<$ Manage flight strips $>$, i.e. $<$ Strip marking $>$ worked as a communication channel, transporting an excess of variability, which played a relevant role for the emergence of the event itself, even considering that there were no chance to interrupt its excess of variability (see the five C-O connections of $<$ Strip marking $>$ in Figure 10). After SKW5569 was authorized to line up on the runway and before USair1493 was authorised to land, the LC2 became aware of the lack of a flight progress strip. This flight progress strip was related to another aircraft, i.e. WW5072 next to ask for taking off. The LC2 did not have the flight progress strip for the fact that people at LAX operated in constancy of an incongruence between the function $<$ Organise operations strategically> and the function < Organise operations tactically>, which directly controls the ATCO’s function (see Figure 10).

More in detail, by June 1988, the National Legislation for Strategic Planning did provide that the Ground Controller (GC) prepares [...] reviews [...] revises [...] marks [...] forwards the flight progress strip to the appropriate (control) position. On the contrary, at the tactical and operational level it was published by January $11^{\text {th }} 1990$, the Supplement 1 to National order $7220.2^{\circ}$. This amendment stated that the strip (has to) be forwarded to the appropriate local control position, but also that there is no strip marking required of ground control. Strip management was considered a coordination item between the Delivery Controller (DC) and LC2 while, at the same time, the aircraft management representing the physical counterpart of flight progress strip, was related to DC, GC and LC2. 


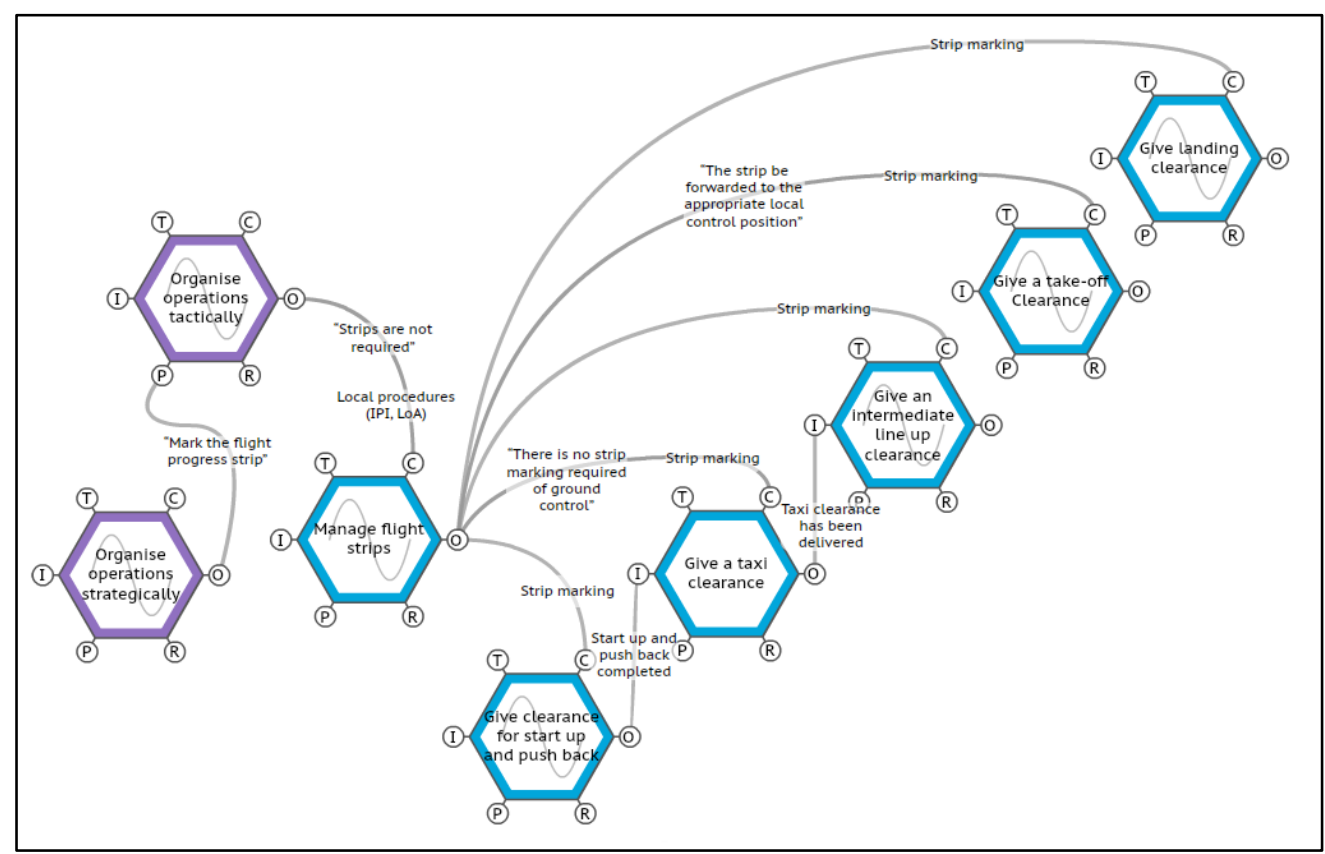

Figure 10. The relevant (red or yellow couplings in terms of criticality) systemic effects of $<$ Manage flight strips $>$ in the USair 1943 accident and respective upstream relevant functions.

In this case, the strategic organizational function was not able to dampen the variability for a different intention of the tactical organization function. Consequently, the operational interpretation by blunt-end operators was misled by middle management (at tactical level) that, in turn, misled the sharp-end operators, in terms of their local rationality. Middle management intended to decrease the GC's workload, not considering the side effects in terms of LC2's increased workload. The analysis also shows how $<$ Strip management>, which is fundamentally an individual function, has strong systemic implications, seriously affecting the system (DL=12) in the operational scenario.

By similar observations, it is then possible to understand the local rationality -rather than finding a unique root cause- of the accident and even develop a proactive safety assessment to properly manage variability. For example, the function <Give a take-off clearance $>$ were a source of variability because in the work-asdone its output were "Imprecise" and "Not in time" (see Figure 8). Subsequently, since the aircrafts were not receiving authorization for take-off, workload for ATCO and pilots progressively increased, causing an abnormal disruptive flow of variability. Consequently, for properly manage this variability in other events, it would be necessary assess more accurately the priority to assign for take-off clearance in order to avoid extra workload due to a congested RWY. Rather than a simple sharp-end barrier, the proactive safety analysis should focus on organizational effects, even considering other aspects to re-iterate the process. It could be interesting to observe that 6 weeks before the event, the function $<$ Give a take-off clearance $>$ was evaluated as part of a comprehensive and periodical assessment, identifying deficiencies that were 
indicative of weaknesses ${ }^{32}$. However, the decision maker failed to dispose an effective action for managing system's variability, lacking of a holistic depiction of the operational scenario, and thus ignoring the systemic effects of a potential disruptive variability. On this path, FRAM by systematic assessments offered by RAM would support this type of observations.

\section{Conclusions}

FRAM allows systemic retrospective (event investigation) and prospective safety analyses (risk assessment). FRAM is one of the methods based on Safety-II and Resilience Engineering, which take into account nowadays systems' complexity.

\section{About other approaches}

FRAM's functional perspective overcomes the traditional causality credo, which is strongly rooted in traditional safety analysis method. For example, the Root Cause Analysis (RCA), relying on the Domino model explains events as developing following a chain reaction of causes and effects where the first domino piece that falls represents the root cause while the last domino piece represents the final outcome or injury 1. Similar reflections emerge when analysing Rasmussen's Accimap, a method to identify causal factors at different management levels that aims to identify the causal relation among events by linking physical sequence of events and activities at governmental, regulatory, and societal levels ${ }^{1}$. A case study in an offshore operation's accident modelling proves the relevance of FRAM with respect to Fault Tree Analysis (FTA) to detect non-linear interactions of various functions, especially in case of complex and vast systems. FRAM is powerful since modelling everyday work does not require operational data of failure, which are rare in high-reliability systems ${ }^{19}$.

When compared to traditional Failure Mode and Effects Analysis (FMEA), FRAM has been assessed to be useful as well. Firstly, it forces consideration of the different contextual aspects that are usually not included in the simple sequential process maps used as a basis for the application of FMEA itself. Secondly, it is advocated to be more intuitive than FMEA, since it does not require consideration of failures and absolute consequences but qualitative assessment of variability, judgments which practitioners perceive more comfortably ${ }^{9}$. FRAM can be considered advantageous even with respect to Hazard and Operability Analysis (HAZOP), since FRAM does not require isolating attributes or information flows, with the inherent potential to mask more complex problems that arise only when multiple attributes or flows interact in complex ways ${ }^{33}$. 
As a general observation, the effectiveness of applying FRAM is related to the characteristics of the system under investigation. If the event concerns the operations of a components or a sequence of components, the investigation can be developed using traditional analysis based on cause-effect structural reasoning (RCA, FTA, FMECA, etc.). Otherwise, if the event takes into account both human and organizational aspects in a broader perspective, it is necessary to acknowledge inherent complexity of the system, using a systemic perspective based on functional approaches. More generally, the advantages of using a combined SafetyI/Safety-II approach has been strongly emphasized for gaining holistic analysis of complex and complicated systems, requiring both a technological and a system-wide focus ${ }^{34,35}$.

\section{About the outcome of the method}

Starting from the positive case studies obtained applying FRAM for systemic accident analysis, this paper explores the possibility of enhancing FRAM's classical formulation in order to generate assessments that are more systematic. As observed in literature ${ }^{27}$, the graphical representation of a FRAM model in case of a complex process may become quickly highly interconnected and thus overwhelming to interpret. This paper, discussing a simple and user-friendly tool, i.e. the RAM, shows the benefits arising from a matrix of functional resonance. RAM allows understanding, in a structured way, the effects of variability and its propagation both upstream and downstream in the system. This approach might lead to identifying critical sources of functional resonance, and thus ways to manage in terms of system's resilience.

RAM is not supposed to be a standalone tool, but it has to be used as a support tool for developing a FRAM model and the standard graphical representation. The illustrative case study based on the LAX accident happened in 1991, details the practical application steps, detailing how a FRAM analysis might guide the observation of an event, linking different process and different actors in a functional perspective.

\section{About future research}

FRAM and RAM could be combined with the model-aided checker to visualize all the actual paths of a FRAM model, as discussed by ${ }^{26}$. Furthermore, RAM perfectly fits with a recent evolution of FRAM addressing a semi-quantitative structure based on Monte Carlo simulation ${ }^{23}$. In this case, it would be possible to define distributions for variability of functions based on SMEs' judgments, historic data, or even observational studies, and then explore the upstream and downstream couplings by a computational algorithm based on the RAM conceptual idea.

On this path, the purpose of the model would consists of creating functional hot spots. In aviation, physical hot spots already exist in those airports where the complexity exceeds the possibility to have a holistic situational awareness. In these scenarios, ATCOs, pilots and ground personnel need to know specific physical positions (called hot spots) where it is more common, more possible, that a runway incursion 
might occur. Therefore, RAM would help answering some questions: Is it be possible to identify functions that represent an abstract hot spot, along the lines of the physical ones? Is it possible to detect a recurring critical coupling, between two or more functions with critical systemic consequences? Is there any "black swan" that can emerge because of systemic variability? The RAM might offer a valuable contribution answering these questions, systematically exploring the potential relationships in a FRAM model.

\section{Acknowledgements}

One of the author (RP) developed a VBA code to execute the analysis conducted in this study and interfaced it with FMV, a software written and developed by Rees Hill, based on prof. Hollnagel studies, which the authors greatly acknowledge for their contributions to the development of FRAM.

\section{References}

1. Hollnagel E. FRAM: The Functional Resonance Analysis Method - Modelling Complex Socio-technical Systems. Ashgate, 2012.

2. $\quad$ EU Commission. Impact assessment of revision to Regulation 95/93. 2011.

3. $\quad$ EU Commission. MEMO/11/857 Europe's Airport 2030: Challenges Ahead. 2011.

4. $\quad$ EUROCONTROL. A White Paper on Resilience Engineering for ATM. 2009.

5. $\quad$ Lundberg J, Woltjer R. The Resilience Analysis Matrix (RAM): Visualizing functional dependencies in complex socio-technical systems. In: Proceedings of the 5th Resilience Engineering Association Symposium. Soesterberg (The Netherlands), 25 - 27 June 2013, 2013.

6. Belmonte F, Schön W, Heurley L, et al. Interdisciplinary safety analysis of complex sociotechnological systems based on the functional resonance accident model: An application to railway trafficsupervision. Reliab Eng Syst Saf 2011; 96: 237-249.

7. Lundblad K, Speziali J, Woltjer R, et al. FRAM as a risk assessment method for nuclear fuel transportation. In: International Conference Working on Safety. Crete, Greece, 2008.

8. Shirali GA, Mohammadfam I, Ebrahimipour V. A new method for quantitative assessment of resilience engineering by PCA and NT approach: A case study in a process industry. Reliab Eng Syst Saf 2013; 119: 88-94.

9. Sujan M, Felici M. Combining failure mode and functional resonance analyses in healthcare settings. Lect Notes Comput Sci (including Subser Lect Notes Artif Intell Lect Notes Bioinformatics) 2012; 7612 LNCS: 364-375.

10. Clay-Williams R, Hounsgaard J, Hollnagel E. Where the rubber meets the road: using FRAM to align work-as-imagined with work-as-done when implementing clinical guidelines. Implement Sci 2015; 10: 125.

11. Pickup L, Atkinson S, Hollnagel E, et al. Blood sampling - Two sides to the story. Appl Ergon 2017; 59: 234-242.

12. Sawaragi T, Horiguchi Y, Hina A. Safety Analysis of Systemic Accidents Triggered by Performance Deviation. In: 2006 SICE-ICASE International Joint Conference. IEEE, pp. 1778-1781.

13. Woltjer R, Hollnagel E. The Alaska Airlines Flight 261 Accident: A Systemic Analysis of Functional Resonance. In: Proceedings of the 14th International Symposium on Aviation Psychology. Antibes Juans les Pins (France), 2007. Epub ahead of print 2007. DOI: 10.1017/CBO9781107415324.004.

14. Woltjer R. Resilience Assessment Based on Models of Functional Resonance. In: 3rd Symposium on Resilience Engineering. 2007, pp. 299-303.

15. Nouvel D, Travadel S, Hollnagel E. Introduction of the Concept of Functional Resonance in the Analysis of a Near-Accident in Aviation. In: 33rd ESReDA Seminar: Future challenges of accident investigation, p. 9 pages.

16. Hollnagel E, Pruchnicki S, Woltjer R, et al. Analysis of Comair flight 5191 with the functional resonance accident model. 8th International Symposium of the Australian Aviation Psychology Association 2008; 8 pages.

17. De Carvalho PVR. The use of Functional Resonance Analysis Method (FRAM) in a mid-air collision to understand some characteristics of the air traffic management system resilience. Reliab Eng Syst Saf 2011; 96: 1482-1498. 
18. Herrera IA, Woltjer R. Comparing a multi-linear (STEP) and systemic (FRAM) method for accident analysis. Reliab Eng Syst Saf 2010; 95: 1269-1275.

19. Toroody AB, Abaei MM, Gholamnia R. Conceptual compression discussion on a multi-linear (FTA) and systematic (FRAM) method in an offshore operation's accident modeling. Int J Occup Saf Ergon 2016; 22: 532-540.

20. Praetorius GG., Hollnagel E. EE. E, Dahlman JJ. Modelling Vessel Traffic Service to understand resilience in everyday operations. Reliab Eng Syst Saf 2015; 141: 10-21.

21. Herrera IA, Hollnagel E, Håbrekke S. Proposing safety performance indicators for helicopter offshore on the Norwegian Continental Shelf. PSAM 10 - Tenth Conference on Probabilistic Safety Assessment and Management 2010; 10.

22. Ragosta M, Martinie C, Palanque P, et al. Concept Maps for Integrating Modeling Techniques for the Analysis and Re-Design of Partly-Autonomous Interactive Systems. In: Proceedings of ATACCS 2015 - 5th International Conference on Application and Theory of Automation in Command and Control Systems 30 Sept-2 Oct. Toulouse (FR), 2015, pp. 41-52.

23. Patriarca R, Di Gravio G, Costantino F. A Monte Carlo evolution of the Functional Resonance Analysis Method (FRAM) to assess performance variability in complex systems. Saf Sci 2017; 91: 49-60.

24. Patriarca R, Di Gravio G, Costantino F, et al. The Functional Resonance Analysis Method for a systemic risk based environmental auditing in a sinter plant : A semi-quantitative approach. Environ Impact Assess Rev 2017; 63: 72-86.

25. Patriarca R, Di Gravio G, Costantino F. Resilience Engineering for space missions' safety assessment. In: IAASS (ed) Proceedings of the 8th IAASS Conference 'Safety First, Safety for all'. Melbourne (Florida): IAASS, 2016, pp. 228-238.

26. Zheng Z, Tian J, Zhao T. Refining operation guidelines with model-checking-aided FRAM to improve manufacturing processes: a case study for aeroengine blade forging. Cogn Technol Work; 18. Epub ahead of print 2016. DOI: 10.1007/s10111-016-0391-1.

27. Patriarca R, Bergström J, Di Gravio G. Defining the Functional Resonance Analysis space : combining Abstraction Hierarchy and FRAM. Reliab Eng Syst Saf 2017; 165: 34-46.

28. Perrow C. Normal Accidents - Living with High-risk Technologies. Harper Collins Publishers, 1984.

29. van Es GWH. Review of Air Traffic Management-related accidents worldwide: 1980 - 2001. 2003.

30. ICAO. Doc 9870 AN/463 - Manual on the Prevention of Runway Incursions. 2007.

31. Cardosi K, Falzarano P, Han S. Pilot-Controller Communication Errors: An Analysis of Aviation Safety Reporting System (ASRS) Reports. 1998.

32. NTSB. Runway Collision of USAir Flight 1493, Boeing 737 and Skywest Flight 5569 Fairchild Metroliner, Los Angeles International Airport. Los Angeles, California, 1991.

33. Frost B, Mo JPT. System Hazard Analysis of a Complex Socio-Technical System: The Functional Resonance Analysis Method in Hazard Identification. In: Australian System Safety Conference 28 30 May 2014. Melbourne Australia, 2014.

34. Hollnagel E. Safety-I and Safety-II (The past and future of Safety Management). Farnham, UK: Ashgate, 2014.

35. Patriarca R, Di Gravio G, Costantino F. Resilience engineering to assess risks for the air traffic management system: A new systemic method. Int J Reliab Saf; 10. Epub ahead of print 2016. DOI: 10.1504/IJRS.2016.10005344. 


\section{Biographies}

\section{Riccardo Patriarca}

BSc in Aerospace Engineering, MSc in Aeronautical Engineering. Currently Ph.D. candidate in Industrial and Management Engineering at Sapienza University of Rome; assistant lecturer of Operations Management, and Quality Management at the Department of Mechanical and Aerospace Engineering (Sapienza University of Rome). His research activity is focused on risk and safety management and resilience engineering in complex socio-technical systems, especially related to aviation and other transportation domains, process plants and healthcare.

\section{Gianluca Del Pinto}

He holds a Bsc in humanities and an Msc in contemporary history. Currently he is an Msc student in human computer interaction. He has been working for ENAV, the Italian ANS Provider, since 2001. After being employed as office worker, he worked firstly as flight information service officer, then as air traffic controller. He currently holds the role of director of communications committee of ANACNA, the Italian Air Traffic Controllers' Association. His current domain of interests is safety, in relation with human factor and non-technical skills, developing processes to manage the performance variability of complex socio-technical systems.

\section{Giulio Di Gravio}

MSc in Mechanical Engineering, Master Degree in Quality Management and Engineering and Ph.D. in Engineering of Industrial Production, he is an assistant professor of Operations Management at the Department of Mechanical and Aerospace Engineering, "Sapienza” University of Rome. His research activity is focused on the analysis and design of industrial, organizational and network systems, information management, performance measurement, risk management and knowledge management, in particular in safety domain of the Air Traffic Management system.

\section{Francesco Costantino}

MSc in Mechanical Engineering, Master Degree in Quality Management and Engineering and Ph.D. in Engineering of Industrial Production, he is an assistant professor of Operations Management at the Department of Mechanical and Aerospace Engineering, "Sapienza” University of Rome. His research activity is focused on the analysis and design of industrial, organizational and network systems, with particular attention to risk management, environment and sustainability, supply chain management, methodologies of improvement (Lean Production and Six Sigma) for organizations. 